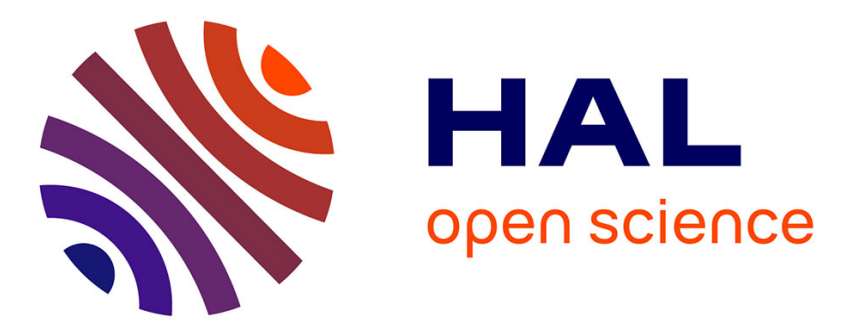

\title{
Relationship between protein structural fluctuations and rebinding dynamics in ferric haem nitrosyls.
}

Neil T Hunt, Gregory M Greetham, Michael Towrie, Anthony W Parker, Nicholas P Tucker

\section{- To cite this version:}

Neil T Hunt, Gregory M Greetham, Michael Towrie, Anthony W Parker, Nicholas P Tucker. Relationship between protein structural fluctuations and rebinding dynamics in ferric haem nitrosyls.. Biochemical Journal, 2011, 433 (3), pp.459-468. 10.1042/BJ20101496 . hal-00558098

\section{HAL Id: hal-00558098 https://hal.science/hal-00558098}

Submitted on 21 Jan 2011

HAL is a multi-disciplinary open access archive for the deposit and dissemination of scientific research documents, whether they are published or not. The documents may come from teaching and research institutions in France or abroad, or from public or private research centers.
L'archive ouverte pluridisciplinaire HAL, est destinée au dépôt et à la diffusion de documents scientifiques de niveau recherche, publiés ou non, émanant des établissements d'enseignement et de recherche français ou étrangers, des laboratoires publics ou privés. 


\title{
Relationship between Protein Structural Fluctuations and Rebinding Dynamics in Ferric Haem Nitrosyls
}

\author{
Neil T. Hunt, ${ }^{1}$ Gregory M. Greetham, ${ }^{2}$ Michael Towrie, ${ }^{2}$ Anthony W. Parker ${ }^{2}$ and Nicholas P. Tucker $^{3 *}$
}

1) Department of Physics, University of Strathclyde, Glasgow, G4 0NG, UK 2) STFC Rutherford Appleton Laboratory, Harwell Science and Innovation Campus, Didcot, Oxon, OX11 0QX, UK 3) Strathclyde Institute for Pharmaceutical and Biomedical Sciences, University of Strathclyde, 161 Cathedral Street, Glasgow, G4 0RE, UK

The interaction of nitric oxide (NO) with haem proteins is widespread in biology. Here, we present the first ultrafast 2D-IR spectroscopic analysis of haem nitrosylation, which has been combined with time-resolved infrared pump-probe studies to investigate the relationship between equilibrium vibrational dynamics of the haem environment and ligand rebinding behaviour following photolysis of $\mathrm{NO}$ from the $\mathrm{Fe}^{\mathrm{III}}-\mathrm{NO}$ site. Studies of two haem proteins, myoglobin $(\mathrm{Mb})$ and cytochrome-c $(\mathrm{Cc})$, which play different physiological roles, reveal marked contrasts in the ultrafast fluctuations of the protein pockets containing the haem, showing that the Mb pocket is somewhat

\section{Introduction}

The structure of biological molecules defines their functionality and specificity. It is also clear that proteins are not static entities but that they undergo changes in structure on a wide range of time and length scales. These changes may take the form of rapid fluctuations, which occur on the order of tens of femtoseconds (fs) to picoseconds (ps) or sidechain motions and secondary structure changes that take place on nanosecond to millisecond timescales. Furthermore, the dynamic effects of hydrogen bonding add an extra layer of complexity. Despite a significant amount of work examining the interplay of structure, dynamics and reactivity however, a clear picture of the role of ultrafast motions in determining protein behaviour has not been established.

Haem proteins have been the subject of much study as a result of their diverse roles in fundamental biological processes[1, 2]. In particular, the dynamics of binding small molecules such as $\mathrm{O}_{2}$ and $\mathrm{CO}$ and the behaviour of these systems following ligand photolysis have attracted significant interest, showing the rebinding kinetics to be a complex function of protein and haem group dynamics [3-10]. Ultrafast vibrational echo [11-13] and 2D-IR spectroscopies have also emerged as an effective probe of these systems [14-16]. The equilibrium fluctuations of a number of carboxyhaem proteins and their mutants have been investigated by exploiting the ability of the $\mathrm{CO}$ ligand to reflect the fluctuations of the haem pocket electrostatic environment due to the protein scaffold [17-20]. By determining the frequency-frequency correlation function (FFCF) for the $\mathrm{CO}$ vibration in combination with molecular dynamics simulations, these studies have revealed protein dynamics on a range of timescales.

The NO radical is of fundamental importance in biology, participating in processes such as vasodilation, signalling and immunity [21, 22]. As a radical, NO is highly reactive with transition metals such as iron, via interactions with their $d$ orbitals [22]. Metalloproteins such as those containing haem groups are therefore particularly sensitive targets for the NO radical. This is particularly notable since some of the most fundamentally important proteins in the respiratory chain and TCA cycle include NO sensitive haem groups and ironsulphur clusters $[23,24]$. Most organisms are able to sense NO and alter gene expression accordingly, allowing that organism to elicit a physiological response or to detoxify harmful quantities of $\mathrm{NO}[25,26]$. To date, studies of the interactions of the NO ligand with haem proteins have concentrated largely on the ferrous form, facilitating useful comparisons with the carboxyhaem derivatives discussed above. Unlike $\mathrm{CO}$ and $\mathrm{O}_{2}$, however, $\mathrm{NO}$ binds to ferric haem centres but studies of such systems are generally restricted to rebinding studies of the NO ligand using FTIR[9] or visible light spectroscopy [8]. An exception to this is the nitrophorins more flexible than that of Cc. This correlates strongly with slower observed photolysis rebinding kinetics of $\mathrm{Mb}-\mathrm{NO}$ versus $\mathrm{Cc}-\mathrm{NO}$ and indicates a direct link between ultrafast fluctuations and biological functionality. Further, this indicates the validity of linear response theories in relation to protein ligand binding. Finally, 2D-IR shows that Cc-NO displays two distinct structural sub sites at room temperature that do not exchange on the timescales accessible via the NO vibrational lifetime.

Key words: Biophysics, ultrafast spectroscopy, 2D-IR spectroscopy, haem proteins, NO sensing

[27], which naturally function in the ferric form and which have also been investigated using ultrafast coherence and Raman methods[28] but there exist no studies applying ultrafast IR or 2D-IR methods to study ferric nitrosylated haem proteins.

Here, we employ ultrafast 2D-IR spectroscopy to probe the vibrational dynamics of the $\mathrm{Fe}^{\mathrm{III}}$-NO site and provide insights into the fluctuations of the haem pocket environment of two proteins, $\mathrm{Mb}$ and $\mathrm{Cc}$. These equilibrium dynamics are compared with the non-equilibrium behaviour of the NO ligand following photolysis from the haem centre, allowing the relationships between protein flexibility and rebinding dynamics to be observed. The strong correlation observed indicates a link between ultrafast dynamics and functionality and establish the validity of linear response theories for ligand binding.

Ferric $\mathrm{Mb}$ is rare in healthy muscle tissue where it is reduced to the ferrous form that is capable of the reversible binding of oxygen that defines its primary function. It is however thought to be generated via the detoxification of NO to nitrate by oxymyoglobin [1]. Cc is a redox active protein that must be in its ferric state in order to receive electrons from ubiquinol Cc-reductase (complex III) prior to transfer to Ccoxidase (complex IV). Cc does not bind molecular oxygen as $\mathrm{Mb}$ does, but both proteins can accommodate $\mathrm{NO}$ at their haem groups [2]. As these proteins play very different physiological roles, with $\mathrm{Mb}$ acting as a ligand reservoir while $\mathrm{Cc}$ is involved in electron transfer processes a comparative study enables insights into the possible influence of ultrafast pocket dynamics on biological functionality.

The haem pockets of $\mathrm{Mb}$ and $\mathrm{Cc}$ are structurally well characterised: the $\mathrm{Mb}$ haem exists in a five-coordinate geometry in the deoxy state, with proximal coordination of the iron via the His93 residue [29, 30], leaving a vacant distal site for diatomic ligand binding. The sixth coordination site can also be occupied by a water molecule, though this is competitively displaced by diatomic ligands of the types discussed above. By contrast, in the absence of exogenous ligands the $\mathrm{Cc}$ distal site is coordinated by a sulfur atom from the distal Met80 methionine while the proximal site is coordinated by the His18 residue, leading to a hexacoordinated haem moiety [31] (Fig 1A\&B). In the presence of NO, the distal residue coordination is replaced by the diatomic ligand [32].

\section{Experimental:}

Preparation of proteins. Lyophilised equine heart $\mathrm{Mb}$ or $\mathrm{Cc}$ (Sigma) were resolubilised $(50 \mathrm{mg} / \mathrm{ml})$ in deuterated phosphate buffer (pH 7.0) and MAHMA NONOate (Noc-9, Sigma) was added to a 3 -fold molar excess to nitrosylate the protein. No significant quantities of insoluble protein were observed in the samples. The fact that the infrared vibrational 
mode specific to the ferric haem nitrosyl NO stretching vibration was employed as a probe for all spectroscopic experiments guards against problems due to the presence of small quantities of oxyMb. Both proteins are known to undergo reductive nitrosylation but the timescales for this were observed to be slow in comparison to those for data collection. To confirm this, the FTIR spectrum of the sample was obtained pre and post laser experiments.

Ultrafast Spectroscopy. The experiments were carried out using the ULTRA spectrometer and the methodology employed has been described elsewhere [33]. Briefly, the tunable IR pump $\left(\sim 10 \mathrm{~cm}^{-1}\right.$ bandwidth $)$ and probe $\left(400 \mathrm{~cm}^{-1}\right.$ bandwidth) pulses used for double resonance 2D-IR spectroscopy were generated by two optical parametric amplifiers (OPA) pumped by synchronised Ti:sapphire based regenerative amplifiers with $p s$ and fs pulse duration respectively. Both OPAs used difference frequency mixing of the signal and idler beams to generate the mid-IR wavelength pulses. For 2D-IR spectra acquisition, the pump frequency was scanned across the region of interest for a fixed pumpprobe time delay and the probe spectrum recorded via a $\mathrm{HgCdTe}$ array detector and spectrometer combination that yielded a spectral resolution of $2 \mathrm{~cm}^{-1}$. The pump pulse trains were chopped at half the laser repetition rate $(10 \mathrm{kHz})$ to facilitate collection of $I_{\text {pump on }}-I_{\text {pump off }}$ difference spectra while optical delay lines controlled relative pulse timings. The pump-probe polarization relationships were set to the magic angle to remove the effects of molecular rotation in all experiments except those to determine the infrared anisotropy, which employed alternating parallel and perpendicular polarization geometries. In all experiments the pump pulse intensities were reduced to prevent line broadening effects. Examples of slices through the 2D-IR spectra obtained in this manner showing the sensitivities and signal to noise ratios obtained are presented in Fig 3 below.

For time-resolved infrared $\left(\mathrm{UV}_{\text {pump }}-\mathrm{IR}_{\text {probe }}\right)$ experiments, the methodology was similar but a third OPA produced the necessary $350 \mathrm{~nm}$ wavelength $\mathrm{UV}_{\text {pump }}$ pulses via fourth harmonic generation. The samples were held between two $\mathrm{CaF}_{2}$ windows separated by a PTFE spacer with a thickness of $100 \mu \mathrm{m}$. For time resolved infrared measurements, the sample was flowed in order to refresh the probed volume.

\section{Results}

The FTIR spectra of ferric Mb-NO and Cc-NO (Fig. 1) both display a peak near $1920 \mathrm{~cm}^{-1}$. The Cc-NO spectrum was also observed to feature a shoulder on the blue edge of the main band. Haem-nitrosylation is well-established under these conditions and the peaks are assigned to $\mathrm{N}-\mathrm{O}$ stretching modes of the $\mathrm{Fe}^{\mathrm{III}} \mathrm{NO}$ complex $[9,10]$. Fitting the spectra to Gaussian lineshape functions revealed that, for $\mathrm{Mb}-\mathrm{NO}$, the spectrum was well-represented by a single function, centred at $1921.0 \mathrm{~cm}^{-1}$ with a width of $\sim 10 \mathrm{~cm}^{-1}$ while for Cc-NO two lineshapes were required to achieve a similar quality of fit. In this case, both functions featured a width of $\sim 10 \mathrm{~cm}^{-1}$ and were centred at 1917.2 and $1927.1 \mathrm{~cm}^{-1}$ respectively. The quantitative fitting results are shown in the FTIR section of Table 1 , where $\mathrm{a}_{\text {gauss }}, \bar{v}_{\text {gauss }}$ and $\sigma_{\text {gauss }}$ represent amplitude, frequency and width parameters respectively and the functions are compared to the spectra in Fig 1 (dashed lines). These results suggest that the ferric $\mathrm{Cc}-\mathrm{NO}$ species exists in two sub sites, a situation that has been observed for Mb-CO and its mutants but has not been reported for ferric Cc-NO [19]. Temperature dependence measurements of the FTIR spectrum of $\mathrm{Cc}-\mathrm{NO}$ were carried out but no substantial changes in the lineshapes or evidence of coalescence were observed up to $333 \mathrm{~K}$. In the remaining discussion, we will refer to these sub sites of $\mathrm{Cc}-\mathrm{NO}$ as $\mathrm{A}_{1}$ (main peak, 1917.2 $\mathrm{cm}^{-1}$ ) and $\mathrm{A}_{2}$ (shoulder, $1927.1 \mathrm{~cm}^{-1}$ ).

Fig $2(\mathrm{a} \& \mathrm{c})$ show ultrafast 2D-IR spectra of Cc-NO at two pump-probe time delays. The spectra show a clear negative peak located on the spectrum diagonal at (probe, pump) = $(1917,1917)$ corresponding to the bleach and stimulated emission of the $A_{1}$ site $v=0-1$ transition, along with a positive peak located at $(1889,1917)$ assignable to the transient absorption arising from the $\mathrm{v}=1-2$ transition.
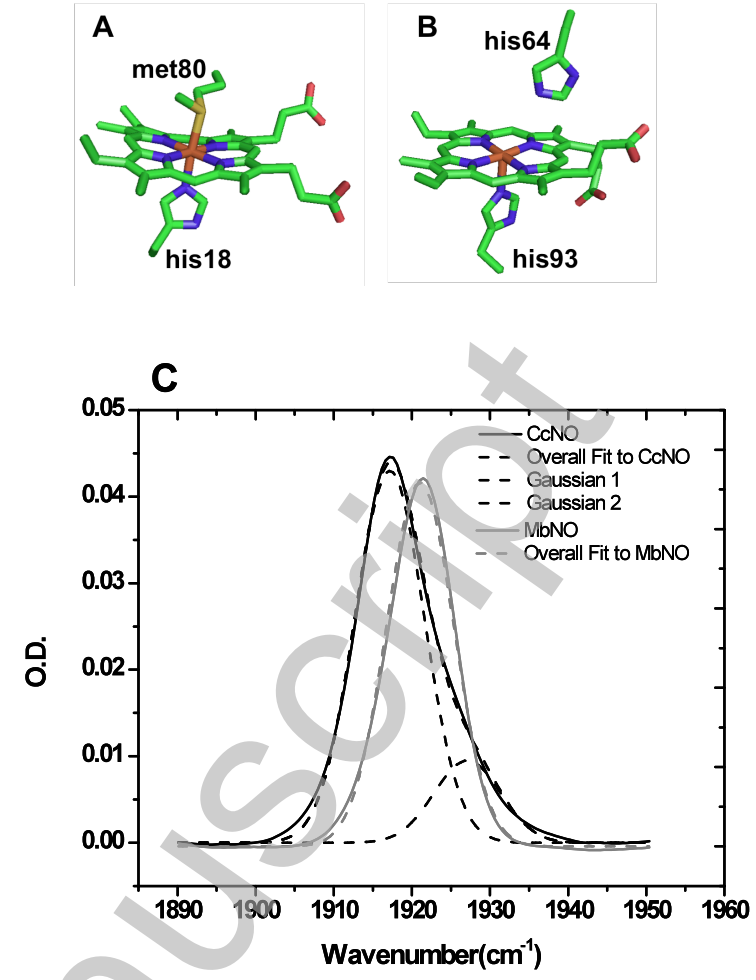

Fig 1: Coordination of the haem groups of equine cytochrome $c(A, P D B$ IHRC[31]) and myoglobin (B, PDB IYMB[30]) demonstrating the structural differences of distal ligands to the ferric haem centre. C) FTIR spectra of ferric Cc-NO (black) and $\mathrm{Mb}-\mathrm{NO}$ (grey) near $1900 \mathrm{~cm}^{-1}$. Solid lines show background-subtracted experimental data while the dotted lines show the results of fitting to Gaussian lineshape functions (see text).

The latter is shifted along the probe axis by $28 \mathrm{~cm}^{-1}$ due to the anharmonicity of the vibrational mode. It is also possible to detect the presence of a much weaker peak pair at (1927, $1927)$ and $(1897,1927)$ that are attributable to the corresponding transitions of the $\mathrm{A}_{2}$ sub site, but these are dominated in the $2 \mathrm{D}$ spectrum by the main $\mathrm{A}_{1}$-derived peaks. The presence of these contributions to the 2D-IR spectra are shown more clearly in Fig 3 using slices through the 2D-IR spectrum taken at a range of pump frequencies for a fixed pump-probe time delay. Fig 3(a) shows slices recorded for pump frequencies to the low frequency side of the centre of the $\mathrm{A}_{1}$ NO stretching mode, while Fig 3(b) shows those for higher pump frequencies. In the latter spectra the presence of a second mode in both the positive $(\mathrm{v}=1-2)$ and negative $(\mathrm{v}=0$ 1) contributions to the spectrum are clearly observed and these are highlighted by arrows in the figure. This is as would be expected because the $A_{2}$ band would only be expected to be present in the slices when the pump frequencies coincided with the spectral envelope of this mode. At the highest pump frequencies, the $\mathrm{A}_{2}$ contribution, though small, is clearly seen to dominate the lineshape. The anharmonic shift of this mode is almost identical to that of the $A_{1}$ sub site.

Fig 4 (a\&c) shows the corresponding 2D-IR spectra for $\mathrm{Mb}$ NO. These consist of a single pair of peaks located near $(1921,1921)$ and $(1893,1921)$ which are attributable to the $\mathrm{v}=0-1$ and $1-2$ transitions of the $\mathrm{N}-\mathrm{O}$ stretching mode of the Mb-NO complex respectively. The effects of anharmonicity are comparable to those observed in the Cc-NO spectra.

In the 2D-IR spectra of $\mathrm{Cc}-\mathrm{NO}$ and $\mathrm{Mb}-\mathrm{NO}$ the lineshapes evolve as a function of pump-probe time delay. This can be perceived in Figs 2 and 4 either as a loss of the diagonal elongation that is present at short pump-probe time delays or as a rotation of the nodal plane between the two components $(v=0-1$ and $v=1-2)$ of the 2D-IR lineshape toward the vertical as the pump-probe time delay increases. This process has been described extensively and is attributed to spectral diffusion of the inhomogeneously-broadened NO vibrational mode $[34,35]$. Inhomogeneous broadening is caused by changes in the electrostatic environment of the NO ligand as the protein pocket fluctuates, which are manifest in the NO 
stretching frequency via a Stark shift of the absorption band. As a result, each NO probe in an ensemble will experience a slightly different environment and thus a slightly different stretching frequency. It has been shown via Stark spectroscopy that the NO stretching frequency provides a sensitive probe of the electrostatic interaction of the protein scaffold with the NO group [36]. Thus, if the protein pocket fluctuations are slow on the timescale of the measurement then a temporal evolution of the 2D-IR lineshape is expected as the NO probes sample each of the environments that contribute to the complete absorption band. When quantified, the temporal evolution of this spectral diffusion provides a measurement of the frequency-frequency correlation function (FFCF), which describes the amplitude and timescale of the ultrafast fluctuations occurring in the haem pocket.

The 2D-IR lineshapes of Mb-NO and Cc-NO obtained at pump-probe delay times from 5 to $50 \mathrm{ps}$ were fit to a $2 \mathrm{D}$ Gaussian function of the form

$$
\left.\sum_{i} A_{i} \exp \left[-\frac{1}{2\left(1-c_{2 D, i}^{2}\right.}\right)\left[\left(\frac{x-x_{0, i}}{\sigma_{x, i}}\right)+\left(\frac{y-y_{0, i}}{\sigma_{y, i}}\right)-\frac{2 c_{2 D, i}\left(x-x_{0, i}\right) y-y_{0, i}}{\sigma_{x, i} \sigma_{y, i}}\right]\right]
$$

where $\mathrm{A}$ represents the amplitude, $\mathrm{x}_{0}$ and $\mathrm{y}_{0}$, are the peak coordinates ( $\mathrm{x}$ represents the probe axis) and $\sigma_{\mathrm{x}}$, and $\sigma_{\mathrm{y}}$, are the corresponding width parameters. The cross correlation parameter, $\mathrm{c}_{2 \mathrm{D}}$, which ranges from -1 to 1 provides a measurement of the diagonal elongation of the lineshape. In the case of $c_{2 D}$, a value of 1 indicates a strongly correlated lineshape elongated along the diagonal of the 2D-IR spectrum. If $c_{2 D}$ is zero, the line will be uncorrelated and circular in shape. Finally, a $c_{2 D}$ value of -1 indicates an anticorrelated lineshape, which will be elongated along the antidiagonal of the spectrum.
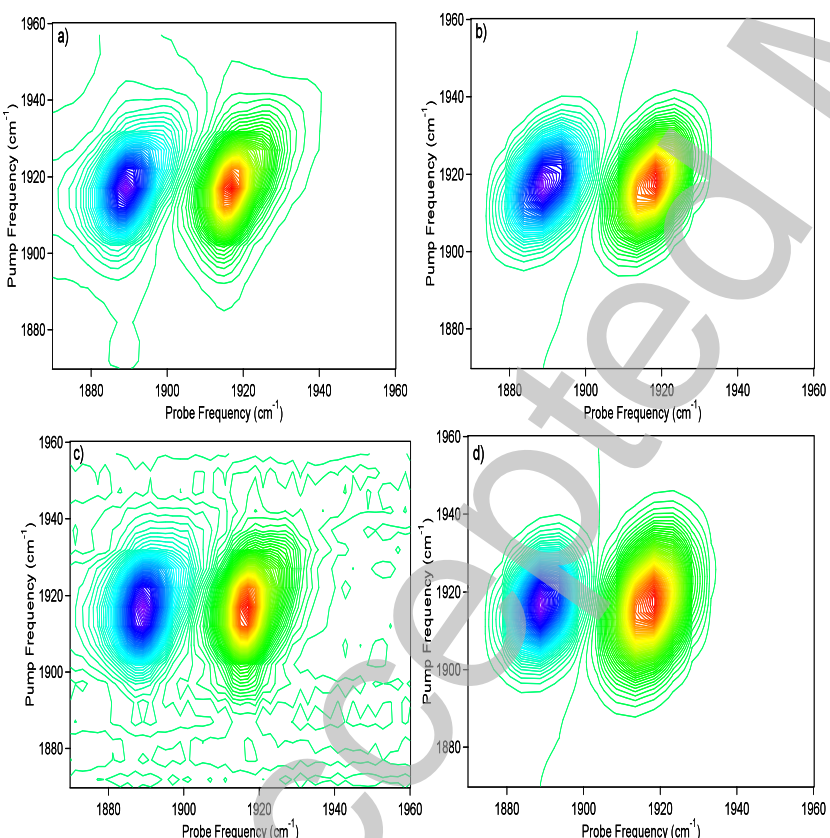

Fig2: 2D-IR spectra of $\mathrm{Cc}-\mathrm{NO}$ recorded with a time delay of a) $5 \mathrm{ps}, \mathrm{c}$ ) $50 \mathrm{ps} . b$ ) and d) are fits to the data in a) and c) respectively using a $2 D$ Gaussian function (see text). Contour color scale runs from red (negative) to blue (positive).

For an inhomogeneously broadened lineshape such as that observed in Figs 2 and 4, the value of c would be expected to vary from a positive value of $c_{2 D}$ towards 0 as the time delay increases due to the NO ligand sampling the different environments created by the haem pocket through protein fluctuation. Quantification of the temporal variation of the $c_{2 D}$ parameter provides the FFCF.

It is noted that the fitting process was started at a pump-probe delay time of 5 ps to avoid coherent contributions to the data caused by temporal overlap of the narrow bandwidth pump pulse with the probe pulse. Fits to the 2D-IR spectra in Figs 2 and 4 are shown in panels (b) and (d) and the temporal variation of $c_{2 D}$ is displayed in Fig 5(a). As the $v=0-1$ and $\mathrm{v}=1-2$ components of the lineshape will be expected to show identical dynamics, the $c_{2 D}$ parameters obtained for each were averaged to provide the data shown in Fig 5(a); the error bars in this figure indicate the repeatability of the data. The lineshape evolution was also analysed using other established metrics [35, 37, 38], which have been shown to yield quantitative measurements of the FFCF, and the dynamics obtained were in excellent agreement with the variation of the $\mathrm{c}_{2 \mathrm{D}}$ parameter. Although the $\mathrm{CC}-\mathrm{NO}$ spectra consist of overlapping lineshapes from two sub sites, the $2 \mathrm{D}$ spectrum was dominated by the $A_{1}$ transitions to such an extent that distortion of the fit by the presence of the shoulder was minimal. This was confirmed by the close agreement of the data derived from the $c_{2 D}$ parameter and the nodal line slope method $[35,38]$ for the $A_{1}$ transitions. The latter uses the rotation of the nodal plane between the two halves of the 2DIR lineshape to quantify the lineshape evolution and so will be unaffected by the presence of the second transition.

Three observations can be made based upon the data in Fig $5(a)$. Firstly, for both proteins, the value of $c_{2 D}$ is less than unity at zero pump-probe time delay. This suggests that some of the processes causing spectral diffusion occur on timescales faster than the $1-2$ ps time resolution of the technique, giving rise to this motionally-narrowed contribution. Secondly, a decay timescale of a few ps is observed for both proteins and thirdly the $\mathrm{c}_{2 \mathrm{D}}$ value does not fall to zero; implying that there are slow, pseudo static processes that lead to spectral diffusion on timescales longer than 50ps. As the measurement is constrained by the vibrational lifetime of the NO ligand (see below) the dynamics of these processes are inaccessible via this experimental methodology.

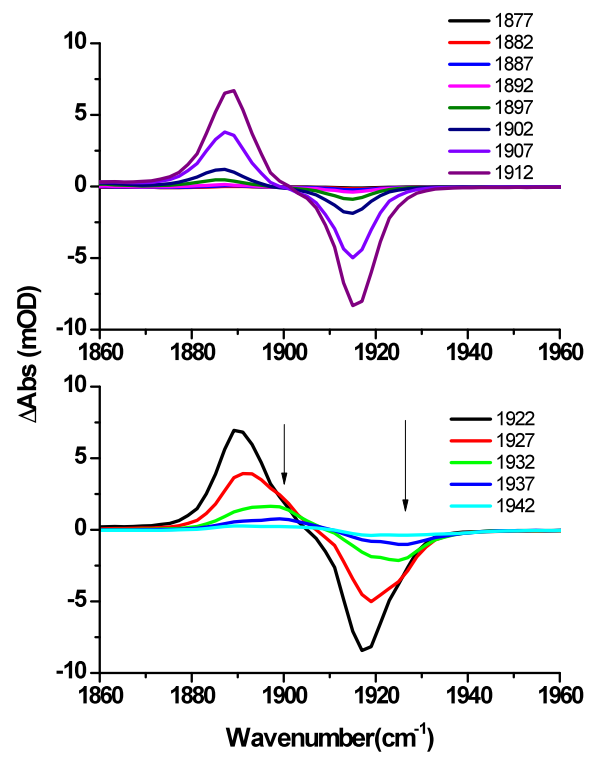

Fig 3: Slices through 2D-IR spectra of CcNO recorded with a pumpprobe delay time of $5 \mathrm{ps}$. (a) Slices corresponding to pump wavelengths on the red side of the main NO stretching transition while $(b)$ shows slices recorded with higher pump frequencies. The legend reports pump frequencies in $\mathrm{cm}^{-1}$. The contributions from the $A_{2}$ state are clearly observed in (b) and are marked with arrows.

The data in Fig 5(a) were fit to the sum of a single exponential decay and a constant term of the form

$$
c_{2 D}=\alpha_{1} \exp \left[\frac{-t}{\tau}\right]+\alpha_{2}
$$


where $\alpha_{1}$ and $\tau$ represent the amplitude and decay time of the exponential function and $\alpha_{2}$ is a constant term; effectively the amplitude of a second decay profile with an infinite decay time. This equation represents a measurement of the FFCF for the NO vibrational mode. The fitting yielded the parameters shown in the Spectral Diffusion section of Table 1. Based upon the fact that the decay time $(\tau)$ is around $5.8 \mathrm{ps}$ for $\mathrm{Mb}$ but $12.3 \mathrm{ps}$ for $\mathrm{Cc}$, the results imply that the protein pocket of $\mathrm{Mb}$ is significantly more flexible than that of $\mathrm{Cc}$. The nondecaying part of the fit is smaller in amplitude for $\mathrm{Mb}$ than Cc, showing that a larger fraction of the possible conformations are sampled during the experimental time window, which is also consistent with increased flexibility.

The vibrational lifetime of the NO stretching mode, the time over which the excited state decays, was obtained from the 2D-IR data and the results for the Cc-NO $\mathrm{A}_{1}$ state are shown in Fig 5(b). The figure shows the decay of the signal from to the v=0-1 and 1-2 transitions which were well-represented by a single exponential function (solid lines) of the form,

$$
\text { PeakAmplitude }=A_{v i b} \exp \left[\frac{-t}{T_{1}}\right]
$$

with a decay time or vibrational lifetime, $\mathrm{T}_{1}$, of around 23 ps (see Vibrational Relaxation section of Table 1, where $\mathrm{A}_{\text {vib }}$ represents the amplitude of the decay function). The results for $\mathrm{Mb}-\mathrm{NO}$ revealed a very similar vibrational lifetime to this state of Cc-NO. Interestingly, the $\mathrm{A}_{2}$ state of $\mathrm{Cc}-\mathrm{NO}$ revealed a somewhat shorter lifetime of $18.5 \mathrm{ps}$ (see Table 1). The dominance of the $2 \mathrm{D}$ signal by the $\mathrm{A}_{1}$ transition made fitting to two 2D Gaussians inconclusive but the two signals were separated using fits of slices (Fig 3) through the 2D-IR data at a pump frequency of $1930 \mathrm{~cm}^{-1}$ to $1 \mathrm{D}$ Gaussian lineshapes. It is important to stress that no evidence of cross peaks linking the $\mathrm{A}_{1}$ and $\mathrm{A}_{2}$ modes were observed, except as a result of pumping the strong $A_{1}$ mode with the wings of the pump pulse bandwidth when the pump frequency was centered on the $\mathrm{A}_{2}$ mode. The reverse peak was not resolved as a result of the relatively low intensity of the $A_{2}$ transition. The lack of these cross peaks implies that the two sub sites are not exchanging on the timescale of the experiment.
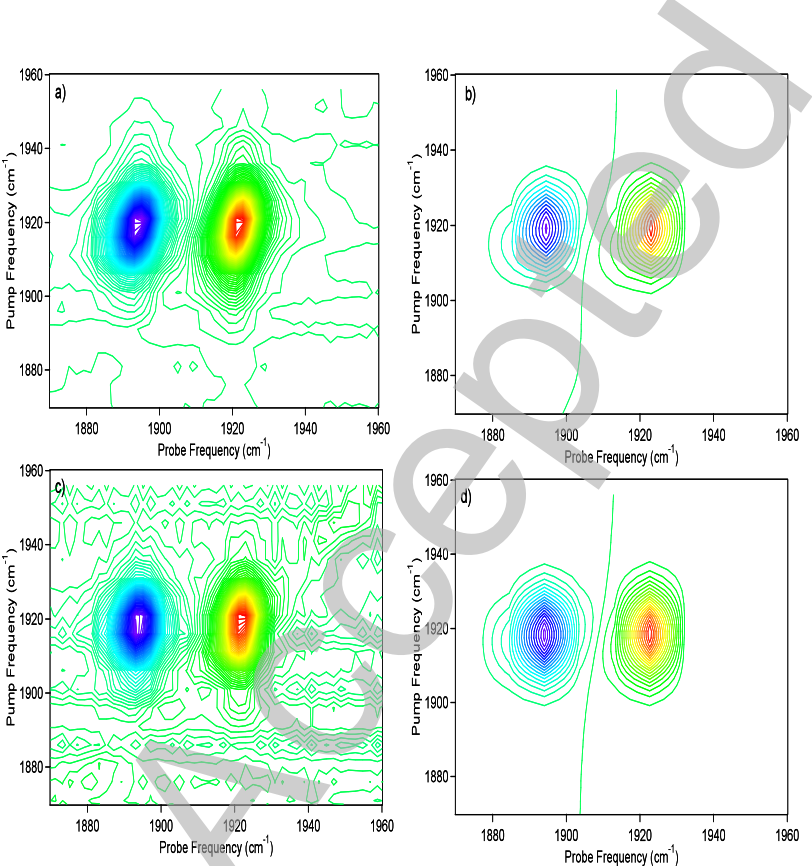

Fig 4: 2D-IR spectra of $\mathrm{Mb}-\mathrm{NO}$ recorded a time delay of a) $5 \mathrm{ps}, \mathrm{c}$ ) $50 \mathrm{ps}$. b) and d) are fits to the data in a) and c) respectively using $a 2 D$ Gaussian function (see text). Contour color scale runs from red (negative) to blue (positive).

Using 2D-IR signals obtained using parallel $\left(\mathrm{R}_{\text {para }}\right)$ and perpendicular $\left(\mathrm{R}_{\text {perp }}\right)$ pump-probe polarization geometries, the rotational relaxation time of the NO (which acts as a probe for the protein) was recovered via the anisotropy decay. The anisotropy parameter is derived from the relation $R_{\text {aniso }}=$ $\left(R_{\text {para }}-R_{\text {perp }}\right) /\left(R_{\text {para }}+2 R_{\text {perp }}\right)$. In order to verify the accuracy of the polarization settings, the magic angle data used for vibrational relaxation measurements were recreated using the relation $R_{M A}=R_{\text {para }}+2 R_{\text {perp }}$, which showed excellent agreement. The temporal dependence of the anisotropy for $\mathrm{Cc}-\mathrm{NO}$ and $\mathrm{Mb}-$ NO were very similar, revealing that the anisotropy of the NO mode undergoes no decay attributable to rotational reorientation, indicating that this process is slow on these timescales, as anticipated for a large protein.

To complement these equilibrium dynamics studies, the behaviour of the Mb-NO and Cc-NO systems following UV photolysis of the NO ligand were determined. The results of the transient infrared measurements (Fig 6) show clear differences between the two proteins. For Cc-NO, photolysis resulted in a bleach of the $A_{1}$ and $A_{2}$ sub sites (Fig 6(a)), with no differentiation between them possible in the observed data either in terms of fractional bleach or recovery rate; no time evolution of the bleach lineshape was observed other than a decrease in amplitude. The dynamics of the recovery (Fig 6(b), black squares) were well-represented by an exponential decay function of the form

PeakAmplitude $=A_{\text {rebind }} \exp \left[\frac{-t}{\tau_{\mathrm{Re} \text { bind }}}\right]+A_{\text {static }}$

where $\mathrm{A}_{\text {rebind }}$ is the amplitude coefficient and $\tau_{\text {Rebind }}$ the timescale for rebinding/recovery respectively; $A_{\text {static }}$ is the amplitude coefficient of the static component, this corresponds to the fraction of NO molecules that do not rebind to the haem within the experimental timescale.
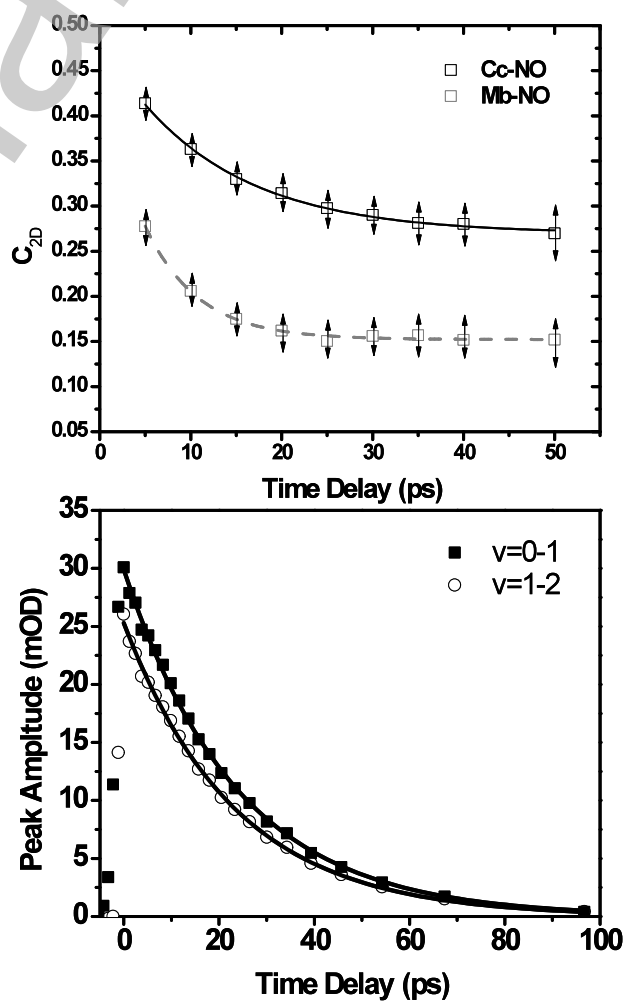

Fig 5: (a) Variation of cross-correlation parameter $c_{2 D}$ as a function of pump probe delay time for $\mathrm{Cc}-\mathrm{NO}$ (black, solid) and $\mathrm{Mb}-\mathrm{NO}$ (grey, dashed). Solid lines show a fit to exponential functions (see text). (b) Vibrational relaxation for Cc-NO. Solid lines represent fits to single exponential decay functions (see text).

The results of the fitting are displayed in the Rebinding Dynamics (bleach) section of Table 1 . For Cc-NO the bleach recovered with a timescale of $12.7 \mathrm{ps}\left(\tau_{\text {Rebind }}\right)$ and a static component $\left(\mathrm{A}_{\text {static }}\right)$ of $\sim 10 \%$ of the initial bleach. Also visible in Fig 6(a) is a small photoproduct peak near $1830 \mathrm{~cm}^{-1}$. 
Fitting the dynamics of this peak to a biexponential function very similar to equation 4 but with two exponential terms with parameters $A_{\text {rise }}$ (amplitude) and $\tau_{\text {rise }}$ (timescale) included to fit the rising portion of the data, $A_{\text {decay }}$ (amplitude) and $\tau_{\text {decay }}$ (timescale) to fit the decay alongside a static amplitude term $\mathrm{A}_{\text {static }}$. This approach gave a rise time $\left(\tau_{\text {rise }}\right)$ of $2.5 \mathrm{ps}$ followed by a 36 ps decay $\left(\tau_{\text {decay }}\right)$ (see Table 1, Photoproduct Dynamics). In the case of the photoproduct, no signal remained at long times, $\left(\mathrm{A}_{\text {static }}=0\right)$ suggesting a transient nature though it is clear that the ground state recovery is likely to be only partially due to direct transfer from the photoproduct given the difference in timescales. The small amplitude of this photoproduct band indicates either a low concentration or a weak transition dipole moment but no significant 36 ps timescale was observed in the bleach recovery.

For Mb-NO photolysis, the recovery timescale for the Mb-NO bleach (Table 1) was also fit to a single exponential function with a static contribution, giving a recovery timescale of $32 \mathrm{ps}$ and a static amplitude of around $30 \%$ of the initial bleach. The initial bleach was an order of magnitude smaller than that obtained for $\mathrm{Cc}-\mathrm{NO}$ and no photoproduct bands were observed. The data for Mb-NO compare well with FTIR studies of ferric Mb-NO rebinding which also indicated a reduced quantum yield in comparison to ferrous $\mathrm{Mb}-\mathrm{NO}$ and an absence of photoproduct absorptions for Fe $\mathrm{Fe}^{\mathrm{III}}-\mathrm{Mb}-\mathrm{NO}$ [9].
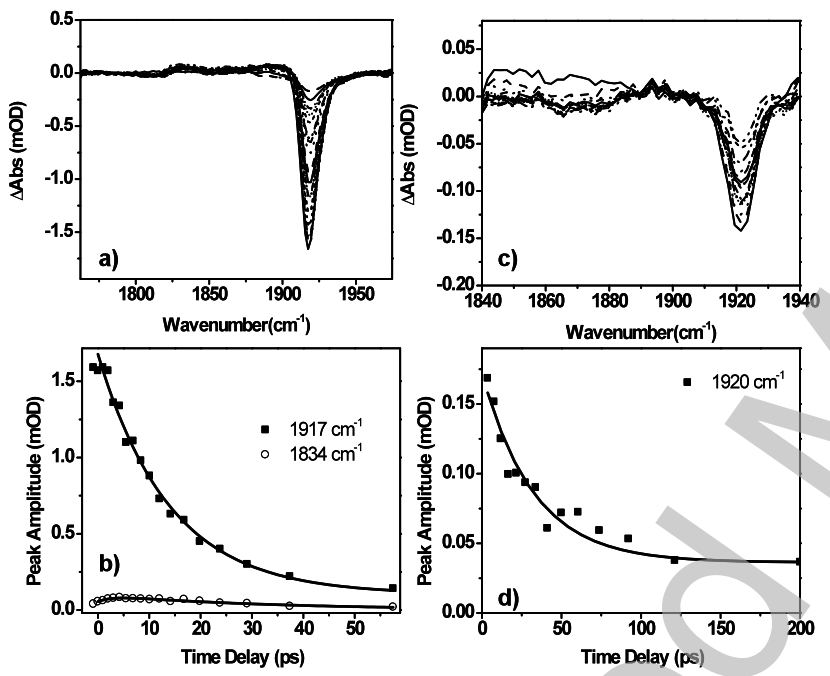

Fig 6: Transient infrared spectroscopy data for NO photolysis from $\mathrm{Cc}$ $N O(a \& b)$ and $M b-N O(c \& d)$. Fits to exponential decay functions in panels $b)$ and $c$ ) are shown as solid lines.

\section{Discussion}

It is clear that the role of the protein structure will be pivotal in determining the observed dynamics. In the NO-bound state, both proteins feature a hexacoordinate $\mathrm{Fe}^{\mathrm{III}}$ centre and a proximally-bound $\mathrm{His}$ residue. In the case of $\mathrm{Mb}$, the distal ligand that is in closest proximity to the NO, and so most likely to participate in any direct protein side-chain-ligand interactions, is the His64 residue (Fig. 1B), while in Cc the equivalent interaction will be via a methionine side chain (Met80) (Fig. 1A). In Cc, two cysteine residues (C14 and $\mathrm{C} 17)$ also bind to the porphyrin ring of the haem group, which may enhance the structural stiffness.

When discussing dynamics, it is also important to consider the binding of the NO to the Fe centre. In the ferric form the $\mathrm{Fe}^{(\mathrm{II})}$ $\mathrm{NO}^{(+)}$description of the ground state $[9,39,40]$ has been shown to be most appropriate, which leads to a ground state that is isoelectronic with that of $\mathrm{Fe}^{\mathrm{II}}-\mathrm{CO}$. The interaction between ligand and the metal centre incorporates backelectron donation between the $\mathrm{Fe}$ and the $\mathrm{N}$ atoms that causes an anticorrelation between the Fe- $\mathrm{N}$ and $\mathrm{N}-\mathrm{O}$ bond strengths and vibrational frequencies. This description also implies a slight positive charge on the NO ligand, which may facilitate interaction with accessible electron lone pairs from nearby distal side chains such as His-64 in the case of Mb (Fig. 1B) $[9,40]$.

Considering the equilibrium dynamics of both proteins, these are broadly similar in that both feature a fast, motionallynarrowed component followed by a ps-timescale decay and a static component that is too slow to be observed via these experiments. Clear differences also occur however, in particular, the FFCF-related parameters obtained for Mb-NO (Fig 5(a)) are smaller in value than those of $\mathrm{Cc}-\mathrm{NO}$, while the ps decay of the function is faster than that of Cc-NO. These point to a faster spectral diffusion rate for the NO ligand in $\mathrm{Mb}$ vs $\mathrm{Cc}$ and thus a more flexible haem pocket environment in $\mathrm{Mb}$.

Comparison of this data with similar studies on wild type ferrous carboxymyoglobin reveals similarities, as expected if the protein scaffold is the significant contributor to the observed dynamics. In particular, a 4.95 ps decay timescale was reported for wild-type $\mathrm{Mb}-\mathrm{CO}$ alongside a static contribution [11-13]. Similar studies of a ferrous carboxy Cc mutant revealed an 8 ps decay time for the FFCF [41], again in good agreement with the parameters observed here for the ferric nitroso forms of Cc. This implies a relative insensitivity of the equilibrium protein dynamics to the oxidation state and ligand identity. 2D-IR investigations of carboxyneuroglobin $(\mathrm{Ngb})$ and mutant $\mathrm{Mb}-\mathrm{CO}$ proteins [19] revealed evidence of motionally-narrowed processes alongside a biexponential decay with 2 and 14 ps timescales and a static component for one subsite $\left(\mathrm{N}_{3}\right)$ and an 11.5 ps exponential decay with a static component for a second sub site $\left(\mathrm{N}_{0}\right)$. The observation of dynamics in the 11-14 ps region indicates similarities with the Cc dynamics observed here. The time resolution of our 2D-IR measurements will result in the faster decay contributions being absorbed into the motionally narrowed dynamical component. In the case of the $\mathrm{H} 64 \mathrm{~V}$ mutant of $\mathrm{MbCO}$, the $\mathrm{CO}$ stretching mode revealed FFCF dynamics on the $5.2 \mathrm{ps}$ timescale [19], which is very close to the value observed for the ferric NO-ligated haem studied here. The H64V mutant lacks any interaction between the distal His residue and the ligand so similarity with wild-type $\mathrm{Mb}-\mathrm{NO}$ may indicate that this residue has little effect on the overall electrostatic fluctuations of the pocket or that effects of the change to the ferric form and the loss of this interaction cancel each other out. However, the L29F mutant of ferrous Mb, which will maintain the distal His interaction, showed a much faster decay profile with 1.7 and 66 ps components[19], indicating that the effects of mutations are likely to be significant. It is highly unlikely however that a single residue will be solely responsible for the dynamics observed.

The observation of a somewhat stiffer protein scaffold in Cc is consistent with NMR studies [42]. As 2D-IR methods are able to access significantly shorter-timescale dynamics than these methods, this suggests the existence of a link between the ultrafast dynamics and the longer timescale processes occurring in a given protein.

The origins of the fluctuations that give rise to these FFCFs are not clear. A Fourier transform relationship exists between the FFCF and the density of vibrational states that contribute to the fluctuations. However, the forms of the FFCF observed in Fig 5(a) suggest that the motions will be of very low (THz) frequency and broad bandwidth. This indicates that, rather than assigning the motions to a single mode or specific group of modes, they must be ascribed to the wide range of collective low frequency librations of protein sidechains or backbone motions that arise from the many degrees of freedom existing in a protein structure. In terms of haem group involvement, no specific evidence for interactions with vibrational modes of the haem are observed, in agreement with previous studies that suggest that haem group motions do not contribute significantly to electric field flutuations on short timescales [11-13].

The structural environment of the NO ligand in these two proteins is interesting, particularly when compared to previous studies on the ferrous carboxy forms. In particular, three binding subsites are observed for $\mathrm{Mb}^{\mathrm{II}}-\mathrm{CO}$ depending on the temperature [11-13] and these have been assigned to different positions of the His64 residue side chain in relation 
to the $\mathrm{CO}$ ligand. These were not observed for $\mathrm{Fe}^{\mathrm{III}}-\mathrm{NO} \mathrm{Mb}$, which showed no evidence for the presence of such sub sites in the FTIR spectrum (Fig 1). It has been suggested that the ferric Mb-NO structure includes an interaction between the non-protonated form of the H64 sidechain and the NO ligand $[9,40]$. The data reported here cannot confirm this interaction except to indicate the presence of only one structural form at room temperature.

\begin{tabular}{|c|c|c|c|c|}
\hline Experiment & Unit & \multicolumn{2}{|c|}{ Cc-NO } & Mb-NO \\
\hline FTIR & & $\mathbf{A}_{1}$ & $\mathbf{A}_{2}$ & \\
\hline $\mathbf{a}_{\text {gauss }}$ (Amplitude) & & 0.043 & 0.010 & 0.041 \\
\hline$\overline{\boldsymbol{v}}_{\text {gauss }}$ (Frequency) & $\mathrm{cm}^{-1}$ & 1917.2 & 1927.1 & 1921.0 \\
\hline $\boldsymbol{\sigma}_{\text {gauss }}$ (Width) & $\mathrm{cm}^{-1}$ & 10.44 & 10.02 & 10.12 \\
\hline Spectral Diffusion & & & & \\
\hline$\alpha_{1}($ Amplitude) & & 0.22 & - & 0.29 \\
\hline $\boldsymbol{\tau}$ (Decay time) & $\mathrm{ps}$ & 12.3 & - & 5.8 \\
\hline $\boldsymbol{\alpha}_{2}$ (Constant term) & & 0.27 & - & 0.15 \\
\hline $\begin{array}{l}\text { Vibrational } \\
\text { Relaxation }\end{array}$ & & & & \\
\hline $\begin{array}{l}\mathbf{A}_{\text {vib }}(\mathrm{v}=1-2) \\
\text { (Amplitude) }\end{array}$ & & $\begin{array}{c}0.030 \\
(0.025)\end{array}$ & $\begin{array}{c}0.002 \\
(0.002)\end{array}$ & $\begin{array}{c}0.015 \\
(0.013)\end{array}$ \\
\hline $\begin{array}{c}T_{1}(v=1-2) \text { (Decay } \\
\text { time })\end{array}$ & $\mathrm{ps}$ & $\begin{array}{c}23.7 \\
(23.3)\end{array}$ & $\begin{array}{c}18.6 \\
(18.1)\end{array}$ & $\begin{array}{l}22.5 \\
(22.5)\end{array}$ \\
\hline $\begin{array}{c}\text { Rebinding } \\
\text { Dynamics (bleach) }\end{array}$ & & & & \\
\hline $\mathbf{A}_{\text {rebind }}$ (Amplitude) & & $1.6 \times 10^{-5}$ & - & $1.4 \times 10^{-4}$ \\
\hline $\begin{array}{l}\boldsymbol{\tau}_{\text {Rebind }}(\text { Rebinding / } \\
\text { recovery time })\end{array}$ & ps & 12.7 & - & 32.4 \\
\hline $\begin{array}{c}\mathbf{A}_{\text {static }} \text { (static } \\
\text { amplitude) }\end{array}$ & & $1.5 \times 10^{-4}$ & & $4 \times 10^{-5}$ \\
\hline $\begin{array}{c}\begin{array}{c}\text { Photoproduct } \\
\text { Dynamics }\end{array} \\
\end{array}$ & & & & \\
\hline $\begin{array}{c}\mathbf{A}_{\text {rise }} \text { (Rise } \\
\text { coefficient) }\end{array}$ & & & & \\
\hline $\boldsymbol{\tau}_{\text {Rise }}$ (Rise time) & $\overline{p s}$ & & & - \\
\hline $\begin{array}{l}\mathbf{A}_{\text {decay }}(\text { Decay } \\
\text { coefficient) }\end{array}$ & & & & \\
\hline $\boldsymbol{\tau}_{\text {decay }}$ (Decay time) & ps & & & \\
\hline $\begin{array}{c}\mathbf{A}_{\text {static }} \text { (static } \\
\text { amplitude) }\end{array}$ & & & & \\
\hline
\end{tabular}

Table 1: Results of fitting 2D-IR and Time Resolved Infrared (TRIR) data (see text).

In the case of $\mathrm{Cc}-\mathrm{NO}$, a second subsite $\left(\mathrm{A}_{2}\right)$ was observed and it is possible to speculate on the structural basis for this. This site shows two main differences to the main $A_{1}$ site in the slightly higher transition frequency and a faster $\mathrm{T}_{1}$ vibrational relaxation time. The higher vibrational frequency of the $A_{2}$ mode implies an increased interaction between the ligand and the protein backbone. This interaction must directly affect the vibrational frequency of the NO ligand, thus an interaction between the NO and the side chains of residues forming the haem pocket are more likely than one between the protein and the haem group. The closest distal ligand is the Met80 residue, which in principle may interact with the NO via the $\mathrm{S}$ atom [40]. Such an interaction would disrupt the backbonding effect between the Fe and the N, leading to a weakened Fe-N bond and a slight strengthening of the $\mathrm{N}-\mathrm{O}$ interaction and increased NO stretching frequency. A secondary effect of this interaction with the protein scaffold would be a faster $\mathrm{T}_{1}$ relaxation. The contact between the ligand and the side chain would provide an alternative pathway for vibrational energy dissipation into the environment, reducing the observed $\mathrm{T}_{1}$ value.

In the case of Cc-NO, no exchange was observed between the two subsites, this is in contrast to observations on $\mathrm{Mb}-\mathrm{CO}$ mutants, which reported an exchange time of $\sim 50 \mathrm{ps}$ between sub sites [17]. The increased stiffness of the Cc pocket would be consistent with a slower exchange time and the lack of temperature dependence of the FTIR spectra reported above also implies a larger barrier to interconversion for $\mathrm{Cc}-\mathrm{NO}$.

Non-equilibrium, ligand rebinding studies of $\mathrm{Mb}-\mathrm{NO}$ and $\mathrm{Cc}-$ NO reveal that both proteins show broadly similar dynamics, with rebinding characterised by a single exponential bleach recovery time. However, the relative timescales are significantly different, with Cc-NO showing a rebinding time that is twice as fast as that of $\mathrm{Mb}-\mathrm{NO}$, while around three times as much $\mathrm{NO}$ escapes the $\mathrm{Mb}$ haem pocket following photolysis as does from $\mathrm{Cc}-\mathrm{NO}$. In addition, $\mathrm{Cc}-\mathrm{NO}$ shows clear evidence of a photoproduct binding site for the NO, while Mb-NO does not. The photoproduct site observed for Cc-NO following photolysis displays a frequency that is similar to those observed for ferrous $\mathrm{Mb}-\mathrm{NO}$ and that of free NO,[43] implying that this species involves NO located in a void in the protein pocket, possibly via H-bonding or dipolar interactions. These sites are likely to be the 'Xenon pockets' that serve as binding sites for $\mathrm{CO}$ following photolysis [44].

The reason for the lower quantum yield for NO photolysis from $\mathrm{Mb}$ is unclear. It is possible that the presence of the nearby His64 residue may play a role in restricting initial migration of the NO ligand that is not matched by the $\mathrm{CcNO}$ Met80 residue. The fact that the Met80 residue coordinates the haem iron while the Mb His64 does not would suggest that this may be an unlikely explanation if mere proximity is the key factor. However, as the His interaction will be through a nitrogen rather than sulfur atom, the distal ligand effect may be expected to be stronger for $\mathrm{Mb}$ than $\mathrm{Cc}$. Indeed, a stronger distal ligand interaction in $\mathrm{Mb}-\mathrm{NO}$ than in $\mathrm{Cc}-\mathrm{NO}$ may explain the single binding geometry observed for $\mathrm{Mb}$ and indicate a His 64 contact in this form while the weaker Met80 interaction in $\mathrm{Cc}-\mathrm{NO}$ results in an equilibrium between subsites with $\left(\mathrm{A}_{2}\right)$ and without $\left(\mathrm{A}_{1}\right)$ distal sidechain interaction. A similar quantum yield was observed for the H64L mutant via FTIR methods [9] and it is interesting to note that once the NO ligand has left the binding site, any increased distal ligand attraction plays no role in speeding up the rebinding dynamics suggesting that this situation is rather complex.

The fact that the dynamics obtained for NO rebinding were described by single exponential functions for both ferric haem proteins is consistent with a study of ferric horseradish peroxidase (hrp), which also reported a single exponential decay profile for geminate NO rebinding [8]. Rebinding studies of the ferric nitrophorins reported biexponential dynamics but these were attributed to closed and open states of the protein. For the closed state, the rebinding time of $15 \mathrm{ps}$ is comparable to that of $\mathrm{Cc}$ while the open form displayed rebinding dynamics an order of magnitude slower [27]. The rebinding dynamics of ferrous $\mathrm{Mb}-\mathrm{NO}$, which display multiexponential characteristics, are significantly more complex than those observed for the ferric form [7]. This indicates that the chemical nature of the Fe-haem centre has a role to play in rebinding processes but not in determining the equilibrium fluctuations of the pocket.

Considering the equilibrium and non-equilibrium studies together shows that the ligand rebinding time for $\mathrm{Mb}$ is $\sim 2.5$ times slower than for $\mathrm{Cc}$. This correlates extremely well with the relationship between the spectral diffusion timescales, which shows a factor of 2.1 faster relaxation for $\mathrm{Mb}$ versus Cc. Together these observations strongly suggest a relationship between pocket stiffness and rebinding dynamics and relate well to the increased percentage of the NO that escapes the pocket, or takes longer than the experimental timescale to rebind, in $\mathrm{Mb}$ versus $\mathrm{Cc}$. It has been speculated that the rebinding timescale for a photolyzed ligand group is directly related to the volume of the protein pocket,[5] this cannot be ruled out though the data presented here also strongly suggest that the dynamics of the protein environment play a significant role.

Such a correlation between the equilibrium dynamics and recovery from a perturbed or non equilibrium state is an important result. Furthermore, it is consistent with the predictions of linear response and fluctuation-dissipation theories[45-47]. These state that the response of a system to a perturbation should be identical to the response to a small 
spontaneous fluctuation about the equilibrium state. The direct correlation between ligand rebinding time and the equilibrium fluctuations observed for these proteins would seem to indicate that these theories apply to protein ligand binding as well as to solute solvent systems. The exact mechanism by which the fluctuations affect the propensity for ligand binding is unclear. It is feasible that a more flexible pocket such as that found in $\mathrm{Mb}$ may restrict movement of the NO ligand back to the binding site following cleavage of the $\mathrm{Fe}-\mathrm{N}$ bond, while the stiffer $\mathrm{Cc}$ pocket does not. The observed photoproduct binding site following $\mathrm{Cc}-\mathrm{NO}$ photolysis suggests a well-defined position for the free ligand while the results for $\mathrm{Mb}$ do not indicate similar behaviour. A second possibility is that the protein fluctuations may alter the structure of the haem porphyrin in such a way as to restrict rebinding. However, no direct evidence for porphyrin ring involvement in the equilibrium dynamics was observed suggesting that any effect is likely to be passively controlled by the protein rather than an active effect of the haem.

These results may also relate to the physiological roles of the proteins, it is reasonable to speculate that a ligand transport and storage protein such as myoglobin would require a pocket that does not restrict simple movement of the ligand on and off the binding site, while the role played by NO in disrupting the electron transport chain suggests that NO will be likely to bind more permanently to $\mathrm{Cc}$. Indeed, the reported $\mathrm{NO}$ off rate tends to be faster for ferric $\mathrm{Mb}$ than for $\mathrm{Cc}$ [2] supporting the conclusion that dynamics play a key role in determining biological function.

In conclusion, the data presented here provide the first insights into the ultrafast equilibrium dynamics of metalloprotein nitrosylation. A combination of $2 \mathrm{D}-\mathrm{IR}$ and time resolved IR methods have been applied to study the structural dynamics and geminate rebinding behaviour of NO bound to two ferric haem proteins, $\mathrm{Cc}$ and $\mathrm{Mb}$. The data suggest that $\mathrm{Cc}-\mathrm{NO}$ has a stiffer protein pocket, with reduced equilibrium fluctuations in comparison to $\mathrm{Mb}$. This correlates well with an increased geminate recombination timescale for $\mathrm{Mb}-\mathrm{NO}$ in comparison to $\mathrm{Cc}-\mathrm{NO}$ and indicates an important role for protein fluctuations in determining ligand binding behaviour in addition to confirming the validity of linear response theories for these systems. Two sub sites of ferric $\mathrm{Cc}-\mathrm{NO}$ are observed, with different vibrational relaxation timescales. These appear to derive from differing interactions between the ligand and the distal Met80 residue though exchange between them was not observed.

Acknowledgments: NTH acknowledges funding from the EPSRC (EP/D071011/1), the European Research Council (202706) and from STFC. AWP, MT and GMG acknowledge STFC and BBSRC funding (FDPG/087)). NPT acknowledges funding from the University of Strathclyde.

\section{References}

1 Brunori, M. (2001) Nitric oxide moves myoglobin centre stage. Trends in Biochemical Sciences. 26, 209-210

2 Cooper, C. E. (1999) Nitric oxide and iron proteins. Biochimica et Biophysica Acta. 1411, 290-309

3 Anfinrud, P. A., Han, C. and Hochstrasser, R. M. (1989) Direct observations of ligand dynamics in hemoglobin by subpicosecond infrared spectroscopy. Proc. Nat. Acad. Sci. U. S. A. 86, 8387-8391

4 Kim, S., Jin, G. and Lim, M. (2004) Dynamics of geminate recombination of NO with myoglobin in aqueous solution probed by femtosecond mid-IR spectroscopy. J. Phys. Chem. B. 108, 20366-20375

5 Carlson, M. L., Regan, R., Elber, R., Haiying, L., Phillips Jr, G. N., Olson, J. S. and Gibson, Q. H. (1994) Nitric oxide recombination to double mutants of myoglobin: role of ligand diffusion in a fluctuating heme pocket. Biochemistry. 33, 10597-10606

6 Zemojtel, T., Rini, M., Heyne, K., Dandekar, T., Nibbering, E. T. J. and Kozlowski, P. M. (2004) NO-bound myoglobin: Structural diversity and dynamics of the NO ligand. J. Am. Chem. Soc. 126, 1930-1931

7 Ye, X., Ionascu, D., Griuia, F., Yu, A., Benabbas, A. and Champion, P. M. (2007) Temperature-dependent heme kinetics with nonexponential binding and barrier relaxation in the absence of protein conformational substates. Proc. Nat. Acad. Sci. U. S. A. 104, 14682-14687

8 Ye, X., Yu, A. and Champion, P. M. (2006) Dynamics of nitric oxide rebinding and escape in horseradish peroxidase. J. Am. Chem. Soc. 128, 1444-1445

9 Miller, L. M., Pedraza, A. J. and Chance, M. R. (1997) Identification of conformational substates involved in nitric oxide binding to ferric and ferrous myoglobin through difference Fourier transform infrared spectroscopy (FTIR). Biochemistry. 36, 12199-12207

10 George, S. J., Allen, J. W. A., Ferguson, S. J. and Thorneley, R. N. F. (2000) Time-resolved infrared spectroscopy reveals a stable ferric heme-NO intermediate in the reaction of Paracoccus pantotrophus cytochrome cd1 nitrite reductase with nitrite. J. Biol. Chem. 275, 33231-33237 11 Merchant, K. A., Noid, W. G., Akiyama, R., Finkelstein, I. J., Goun, A., McClain, B. L., Loring, R. F. and Fayer, M. D. (2003) Myoglobin-CO Substate Structures and Dynamics: Multidimensional Vibrational Echoes and Molecular Dynamics Simulations. J. Am. Chem. Soc. 125, 13804-13818

12 Merchant, K. A., Thompson, D. E., Xu, Q.-H., Williams, R. B., Loring, R. F. and Fayer, M. D. (2002) Myoglobin-CO Conformational Substate Dynamics: 2D Vibrational Echoes and MD Simulations. Biophys. J. 82, 3277-3288

13 Merchant, K. A., Noid, W. G., Thompson, D. E., Akiyama, R., Loring, R. F. and Fayer, M. D. (2003) Structural Assignments and Dynamics of the A Substates of MbCO: Spectrally Resolved

Vibrational Echo Experiments and Molecular Dynamics Simulations. J. Phys. Chem. B. 107, 4-7

14 Hochstrasser, R. M. (2007) Two-dimensional spectroscopy at infrared and optical frequencies. Proc. Nat. Acad. Sci. U. S. A. 104, 14190

15 Ganim, Z., Chung, H. S., Smith, A. W., Deflores, L. P., Jones, K. C. and Tokmakoff, A. (2008) Amide I TwoDimensional Infrared Spectroscopy of Proteins. Accts. Chem. Res. 41, 432-441

16 Hunt, N. T. (2009) Ultrafast 2D-IR spectroscopy applications to biomolecules. Chem. Soc. Rev. 38, 1837-1848 17 Ishikawa, H., Kwak, K., Chung, J. K., Kim, S. and Fayer, M. D. (2008) Direct observation of fast protein conformational switching. Proc. Nat. Acad. Sci. U. S. A. 105, $8619-8624$

18 Ishikawa, H., Kim, S., Kwak, K., Wakasugi, K. and Fayer, M. D. (2007) Disulfide bond influence on protein structural dynamics probed with 2D-IR vibrational echo spectroscopy. Proc. Nat. Acad. Sci. U. S. A. 104, 1930919314

19 Ishikawa, H., Finkelstein, I. J., Kim, S., Kwak, K., Chung, J. K., Wakasugi, K., Massari, A. M. and Fayer, M. D. (2007) Neuroglobin dynamics observed with ultrafast 2D-IR vibrational echo spectroscopy. Proc. Nat. Acad. Sci. U. S. A. 104, 16116-16121

20 Finkelstein, I. J., Ishikawa, H., Kim, S., Massari, A. M. and Fayer, M. D. (2007) Substrate binding and protein conformational dynamics measured by 2D-IR vibrational echo spectroscopy. Proc. Nat. Acad. Sci. U. S. A. 104, 26372642 
21 Alderton, W. K., Cooper, C. E. and Knowles, R. G. (2001) Nitric oxide synthases: structure, function and inhibition. Biochem. J. 357, 593-615

22 Hill, B. G., Dranka, B. P., Bailey, S. M., Lancaster, J. R., Jr. and Darley-Usmar, V. M. (2010) What part of NO don't you understand? Some answers to the cardinal questions in nitric oxide biology. J. Biol. Chem. 285, 19699-19704

23 Justino, M. C., Almeida, C. C., Teixeira, M. and Saraiva, L. M. (2007) Escherichia coli di-iron YtfE protein is necessary for the repair of stress-damaged iron-sulfur clusters. J. Biol. Chem. 282, 10352-10359

24 Mason, M. G., Holladay, R. S., Nicholls, P., Shepherd, M. and Cooper, C. E. (2008) A quantitative approach to nitric oxide inhibition of terminal oxidases of the respiratory chain. Methods Enzymol. 437, 135-159

25 Aono, S. (2008) Metal-containing sensor proteins sensing diatomic gas molecules. Dalton Trans., 3137-3146

26 Tucker, N. P., Le Brun, N. E., Dixon, R. and Hutchings, M. I. (2010) There's NO stopping NsrR, a global regulator of the bacterial NO stress response. Trends in Microbiology. 18, 149-156

27 Benabbas, A., Ye, X., Kubo, M., Zhang, Z. Y., Maes, E. M., Montfort, W. R. and Champion, P. M. (2010) Ultrafast Dynamics of Diatomic Ligand Binding to Nitrophorin 4. J. Am. Chem. Soc. 132, 2811-2820

28 Kubo, M., Gruia, F., Benabbas, A., Barabanschikov, A., Montfort, W. R., Maes, E. M. and Champion, P. M. (2008) Low-frequency mode activity of heme: Femtosecond coherence spectroscopy of iron porphine halides and nitrophorin. J. Am. Chem. Soc. 130, 9800-9811

29 Copeland, D. M., Soares, A. S., West, A. H. and Richter-Addo, G. B. (2006) Crystal structures of the nitrite and nitric oxide complexes of horse heart myoglobin. J. Inorg. Biochem. 100, 1413-1425

30 Evans, S. V. and Brayer, G. D. (1990) Highresolution study of the three-dimensional structure of horse heart metmyoglobin. J. Mol. Biol. 213, 885-897

31 Bushnell, G. W., Louie, G. V. and Brayer, G. D. (1990) High-resolution three-dimensional structure of horse heart cytochrome c. J. Mol. Biol. 214, 585-595

32 Kim, J., Park, J., Lee, T. and Lim, M. (2009) Dynamics of ultrafast rebinding of $\mathrm{CO}$ to carboxymethyl cytochrome c. J. Phys. Chem. B. 113, 260-266

33 Kania, R., Stewart, A. I., Clark, I. P., Greetham, G. M., Parker, A. W., Towrie, M. and Hunt, N. T. (2010) Investigating the Vibrational Dynamics of a 17eMetallocarbonyl Intermediate using Ultrafast Two Dimensional Infrared Spectroscopy. Phys. Chem. Chem. Phys. 12, 1051-1063

34 Kwak, K., Park, S., Finkelstein, I. J. and Fayer, M. D. (2007) Frequency-frequency correlation functions and apodization in two-dimensional infrared vibrational echo spectroscopy: A new approach. J. Chem. Phys. 127, 124503

35 Roberts, S. T., Loparo, J. J. and Tokmakoff, A. (2006) Characterization of spectral diffusion from twodimensional line shapes. J. Chem. Phys. 125, 084502

36 Park, E. S., Thomas, M. R. and Boxer, S. G. (2000) Vibrational Stark spectroscopy of NO bound to heme: effects of protein electrostatic fields on the NO stretch frequency. J. Am. Chem. Soc. 2000, 12297-12303

37 Park, S., Kwak, K. and Fayer, M. D. (2007) Ultrafast 2D-IR vibrational echo spectroscopy: a probe of molecular dynamics. Laser Phys. Lett. 4, 704-718

38 Kwac, K. and Cho, M. (2003) Two-Color Pump-Probe Spectroscopies of Two- and Three-Level
Systems: 2-Dimensional Line Shapes and Solvation Dynamics. J. Phys. Chem. A. 107, 5903-5912

39 Nutt, D. R. and Meuwly, M. (2007) Ferric and ferrous heme iron in nitroso-myoglobin: Computer simulations of stable and metastable states and their infrared spectra. ChemPhysChem. 8, 527-536

40 Soldatova, A. V., Ibrahim, M., Olson, J. S., Czernuszewicz, R. S. and Spiro, T. G. (2010) New light on $\mathrm{NO}$ bonding in $\mathrm{Fe}(\mathrm{III})$ heme proteins from resonance Raman spectroscopy and DFT modelling. J. Am. Chem. Soc. 132, 4614-4625

41 Kim, S., Chung, J. K., Kwak, K., Bowman, S. E. J., Bren, K. L., Bagchi, B. and Fayer, M. D. (2008) Native and Unfolded Cytochrome csComparison of Dynamics using 2DIR Vibrational Echo Spectroscopy. J. Phys. Chem. B. 112, 10054-10063

42 Flynn, P. F., Bieber Urbauer, R. J., Zhang, H., Lee, A. L. and Wand, A. J. (2001) Main chain and side chain dynamics of a heme protein: N-15 and H-2 NMR relaxation studies of R. capsulatus ferrocytochrome c(2). Biochemistry. 40, 6559-6569

43 Kim, S. and Lim, M. (2005) Protein conformationinduced modulation of ligand binding kinetics: A femtosecond mid-IR study of Nitric oxide binding trajectories in Myoglobin. J. Am. Chem. Soc. 127, 8908-8909

44 Tilton, R. F., Kuntz, I. D. and Petsko, G. A. (1984) Cavities in proteins: structure of a metmyoglobin-xenon complex solved to 1.9-A. Biochemistry. 23, 2849-2857

45 Stratt, R. M. and Maroncelli, M. (1996) Nonreactive Dynamics in Solution: The Emerging Molecular View of Solvation Dynamics and Vibrational Relaxation. Journal of Physical Chemistry. 100, 12981-12996

46 Geissler, P. L. and Chandler, D. (2000) Importance sampling and theory of nonequilibrium solvation dynamics in water. J. Chem. Phys. 113, 9759

47 Tao, G. H. and Stratt, R. M. (2006) The molecular origins of nonlinear response in solute energy relaxation: The example of high-energy rotational relaxation. J. Chem. Phys. 125, 114501 